This is a self-archived version of an original article. This version may differ from the original in pagination and typographic details.

Author(s): Conley, Kevin M.; Nayyar, Neha; Rossi, Tuomas P.; Kuisma, Mikael; Turkowski,
Volodymyr; Puska, Martti J.; Rahman, Talat S.

Title: Plasmon Excitations in Mixed Metallic Nanoarrays

Year: 2019

Version: Accepted version (Final draft)

Copyright: @ 2019 American Chemical Society

Rights: In Copyright

Rights url: http://rightsstatements.org/page/lnC/1.0/?language=en

Please cite the original version:

Conley, K. M., Nayyar, N., Rossi, T. P., Kuisma, M., Turkowski, V., Puska, M. J., \& Rahman, T. S. (2019). Plasmon Excitations in Mixed Metallic Nanoarrays. ACS Nano, 13(5), 5344-5355. https://doi.org/10.1021/acsnano.8b09826 


\section{Article}

\section{Plasmon Excitations in Mixed Metallic Nanoarrays}

Kevin M. Conley, Neha Nayyar, Tuomas P. Rossi, Mikael Kuisma, Volodymyr Turkowski, Martti J. Puska, and Talat S. Rahman

ACS Nano, Just Accepted Manuscript • DOI: 10.1021/acsnano.8b09826 • Publication Date (Web): 11 Apr 2019

Downloaded from http://pubs.acs.org on April 12, 2019

\section{Just Accepted}

"Just Accepted" manuscripts have been peer-reviewed and accepted for publication. They are posted online prior to technical editing, formatting for publication and author proofing. The American Chemical Society provides "Just Accepted" as a service to the research community to expedite the dissemination of scientific material as soon as possible after acceptance. "Just Accepted" manuscripts appear in full in PDF format accompanied by an HTML abstract. "Just Accepted" manuscripts have been fully peer reviewed, but should not be considered the official version of record. They are citable by the Digital Object Identifier (DOI®). "Just Accepted" is an optional service offered to authors. Therefore, the "Just Accepted" Web site may not include all articles that will be published in the journal. After a manuscript is technically edited and formatted, it will be removed from the "Just Accepted" Web site and published as an ASAP article. Note that technical editing may introduce minor changes to the manuscript text and/or graphics which could affect content, and all legal disclaimers and ethical guidelines that apply to the journal pertain. ACS cannot be held responsible for errors or consequences arising from the use of information contained in these "Just Accepted" manuscripts. 


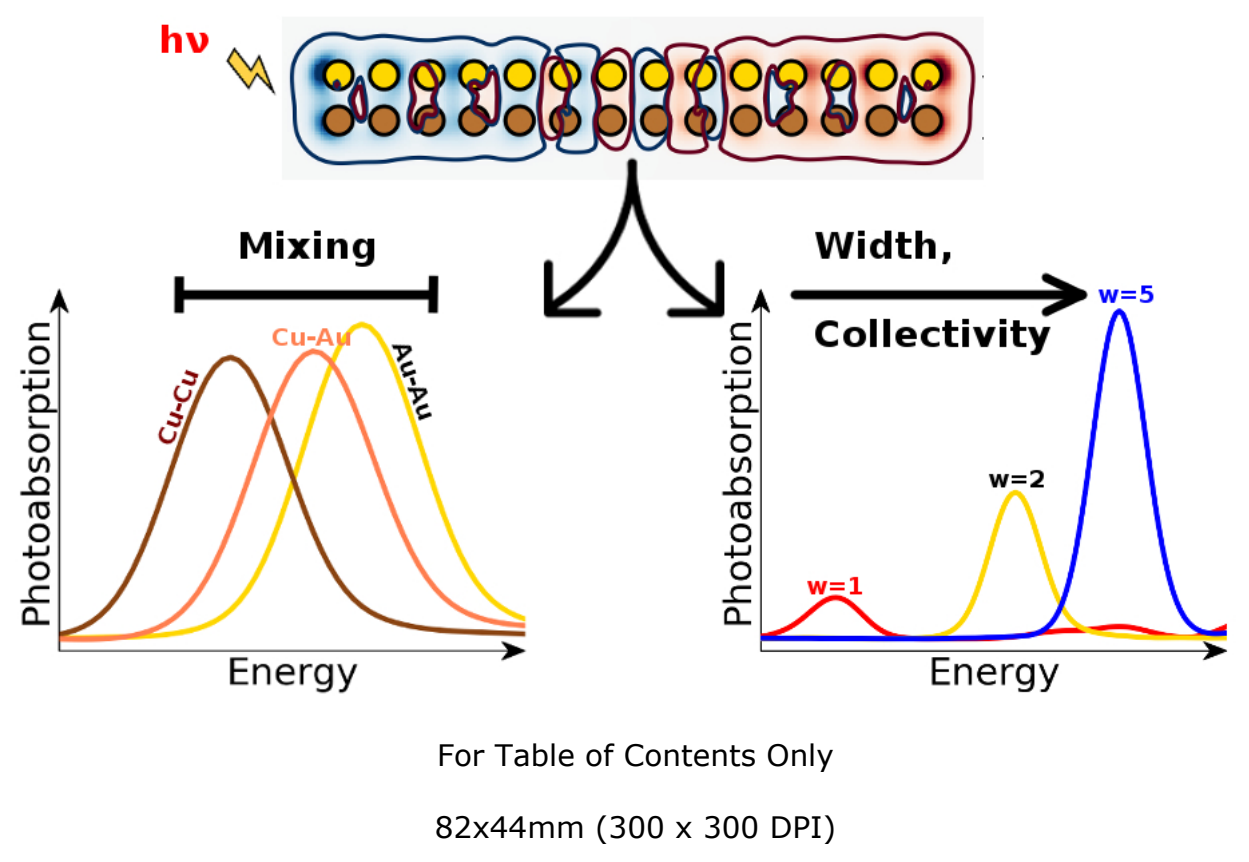

ACS Paragon Plus Environment 


\title{
Plasmon Excitations in Mixed Metallic
}

\section{Nanoarrays}

\author{
Kevin M. Conley, ${ }^{\dagger, \ddagger}$ Neha Nayyar,, I Tuomas P. Rossi, ${ }^{\dagger} \S$ Mikael Kuisma, \\ Volodymyr Turkowski, ${ }^{\text {II }}$ Martti J. Puska, ${ }^{\dagger}$ and Talat S. Rahman*,II, $†$ \\ $\dagger$ Department of Applied Physics, COMP Centre of Excellence, Aalto University School of \\ Science, P.O. Box 11100, FI-00076 Aalto, Finland \\ $\ddagger$ Department of Applied Physics, QTF Centre of Excellence, Aalto University School of Science, \\ P.O. Box 11100, FI-00076 Aalto, Finland \\ IIDepartment of Physics, University of Central Florida, Orlando, Florida, USA \\ §Department of Physics, Chalmers University of Technology, SE-412 96 Gothenburg, Sweden \\ ||Department of Chemistry, Nanoscience Center, University of Jyväskylä, FI-40014 Jyväskylä, \\ Finland \\ E-mail: talat.rahman@ucf.edu
}

\begin{abstract}
Features of the surface plasmon from macroscopic materials emerge in molecular systems, but differentiating collective excitations from single-particle excitations in molecular systems remains elusive. The rich interactions between single-particle electron-hole and collective electron excitations produce phenomena related to the chemical physics aspects within the atomic array. We study the plasmonic properties of atomic arrays of noble $(\mathrm{Au}, \mathrm{Ag}$, and $\mathrm{Cu})$ and transition metal $(\mathrm{Pd}, \mathrm{Pt})$ homonuclear chains using time-dependent density-functional theory and their Kohn-Sham transition contributions. The response to the electromagnetic radiation is related to both the


geometry-dependent confinement of sp-valence electrons, and the energy position of d-electrons in the different atomic species and the hybridization between $\mathrm{d}$ and sp electrons. It is possible to tune the position of the plasmon resonance, split it into several peaks, and eventually achieve broadband absorption of radiation. Arrays of mixed noble and transition metal chains may have strongly attenuated plasmonic behavior. The collective nature of the excitations is ascertained using their Kohn-Sham transition contributions. To manipulate the plasmonic response and achieve the desired properties for broad applications, it is vital to understand the origins of these phenomena in atomic chains and their arrays.

\section{Keywords}

plasmonics, molecular plasmonics, time-dependent density-functional theory, transition contribution maps, collective excitation

The optical properties of nanosystems are highly sensitive to their size, shape, and the chemical composition, and can dramatically differ from those of their bulk cousins. Over the past decade, a wide variety of plasmonic structures based on gold and silver have been fabricated to manipulate light absorption at the nanometer scale. ${ }^{1-4}$ Potential applications rely to some extent on the ability to tune the nanoparticle plasmon resonances, which has played a crucial role in stimulating the current interest in nanoplasmonics. In order to be able to intelligently tune the system properties, including the absorption spectrum, it is essential to understand its dependencies on the physical and chemical parameters.

An early theoretical study on the collective excitations in a few-atom sodium clusters was carried out using Time-Dependent Density-Functional Theory (TD-DFT) ${ }^{5}$ by Kummel et al. ${ }^{6}$ who showed that collective excitations exist even for very small clusters. More recent work addresses the mechanism of the collective excitations and field enhancement in higher dimension 
clusters using TD-DFT. ${ }^{7-9}$ For example, Ma et al. studied the sensitivity of plasmon resonance in Au nanoparticles and their dimers as a function of the particle size and the inter-particle distance. ${ }^{10}$ Studies of the plasmon excitations in two-dimensional planar Na structures ${ }^{11,12}$ or $\mathrm{MoS}_{2}$ nanoflakes ${ }^{13}$ reveal the importance of dimensionality in the formation and development of the plasmon peaks.

Scanning Tunneling Microscope (STM) experiments by Nilius et al. ${ }^{14}$ demonstrated the development of one-dimensional band structure in Au chains on $\mathrm{NiAl}(110)$ when the number of atoms in the chain exceeds 10. Inspired by this experimental finding, theoretical calculations have also predicted the presence of collective plasmon modes in a few-atom chains of several metallic elements: $\mathrm{Na},{ }^{15} \mathrm{Ag},{ }^{16}$ and $\mathrm{Au},{ }^{17}$ and see, e.g., Ref. ${ }^{18}$ for an overview. In a previous study of some of the present authors, ${ }^{19}$ the role of transition metal (TM) doping in the generation of plasmon modes in single Au chains was examined. It was found that doping leads to several additional excitations in the absorption spectrum reflecting changes in the potential around the TM atom and collective effects of the "localized" (TM d-) and delocalized (s) electrons. Indeed, the mutual effects of both electronic subsystems may be nontrivial, in particular leading to a change of the spectral function of the localized electrons ${ }^{20}$ or to local electronic resonances around the dopant atom, as was shown experimentally in the case of Pd-doped Au chains on $\mathrm{NiAl}(110) .{ }^{21}$ This complexity provides an opportunity to tune the optical properties by combining noble and transition metal nanostructures.

To date, there has been a lively discussion on whether the strong absorption modes in small nanosystems or molecules are plasmons or whether they are single-particle excitations. ${ }^{8,22-31}$ In small systems, the plasmonicity of an absorption mode is not sufficiently captured by its collectivity, or the number of contributing electron-hole transitions. For example, the strong absorption peak of atomic Au chains consists of only one Kohn-Sham (KS) transition which lead to the conclusion that the excitation is single-particle-like in nature. ${ }^{24}$ However, Bernadotte et al. ${ }^{25}$ showed that plasmons and single-particle excitations can be differentiated using their dependence on the Coulomb interaction scaling parameter, $\lambda$, in TD-DFT. ${ }^{25}$ Whereas the excitation energy of single particle excitations is largely independent of $\lambda$, plasmons are affected by the Coulomb interaction. 
Using this, it was concluded using that the strong absorption peaks in atomic Au chains around 1 to $2 \mathrm{eV}$ are plasmonic excitations. ${ }^{25}$

The line between plasmonicity and collectivity is further muddled if there is mixing between plasmonic and single-particle excitations or they happen to occur at comparable energies, and some effort has been made to characterize plasmons in molecular systems. ${ }^{24-35}$ The Generalized Plasmonicity Index (GPI) has emerged as a metric both to identify and quantify the plasmonicity of electronic excitations and photonic modes using the strength of the Coulomb interaction of the transitions. ${ }^{31}$ Transition Contribution Maps (TCMs) are another method to identify how the individual KS transitions collectively contribute to the given photoabsorption peaks. ${ }^{36,37}$ The great value of TCMs is that they visualize clearly whether there are low-energy electron-hole transitions which form a plasmonic excitation via Coulomb interaction. ${ }^{25,38}$

While features of plasmons from macroscopic materials are known to emerge in molecular systems, ${ }^{26}$ these systems are far from the size of nanoparticles showing a clear surface plasmon resonance with induced densities restricted on the particle surface. ${ }^{31}$ The intense absorption modes of collective excitations in molecular systems could be described as molecular plasmons to distinguish them from conventional plasmons. However, for short we use the term plasmon interchangeably.

In the present study, we address some of the lingering fundamental questions about the nature of collective excitations in molecular plasmons by considering an arrangement of small atomic chains and arrays. The optical properties of both pure and "mixed" arrays of free-standing homonuclear chains are calculated with time-propagation TD-DFT. ${ }^{39}$ The plasmon character in finite systems such as polyenes ${ }^{27}$ and polycyclic aromatic hydrocarbons ${ }^{40-42}$ has been described previously, and metallic arrays of varying size and composition clearly demonstrate the growing collectivity within molecular plasmons and how to distinguish single-particle and plasmonic excitations. In addition to analyzing the excitation character using TCMs, we show the molecular plasmons are strongly influenced by the interplay between the physical (geometry-dependent electron confinement) and chemical (element-dependent electronic structure) effects within the array. 


\section{Results and Discussion}

In this Section, the optical responses of pure and mixed arrays comprising noble $(\mathrm{Au}, \mathrm{Ag}, \mathrm{Cu})$ or transition $(\mathrm{Pd}, \mathrm{Pt})$ metal atoms are considered using time propagation TD-DFT method with the GLLB-SC exchange-correlation potential ${ }^{43}$ implemented in GPAW ${ }^{38,44}$ (see the Methods section for more details). The effects of the finite size and $s(\mathrm{p})$-d hybridization of electronic states between similar and different types of atoms on the valence electron structure and thereby on the plasmonic response will be discussed.

\section{Molecular plasmons in pure arrays}

Previous studies of single Au chains revealed that increasing the chain length above approximately 8 to 10 atoms generates a strong absorption mode. ${ }^{17,19,24}$ Increasing the number of atoms further intensifies the modes and causes a slowly saturating redshift. In this work, we studied similar trends in the strong absorption mode generated by longitudinal plasmons along the long wire axis in Au nanoarrays. The arrays consist of chains 14 atoms in length and bond length of $2.89 \AA$. The absorption spectra for the single chain of $14 \mathrm{Au}$ atoms as well as for arrays of $n$ chains of $14 \mathrm{Au}$ atoms, where $n=2-5$, are shown in Figure 1A. All of the arrays are planar ( $1 \mathrm{x} n \mathrm{x} 14)$ with the exception of the rod-like $2 \times 2 \times 14$ nanowire. For brevity, planar arrays are denoted $n \times 14$. As examples of the atomic structures, we show the $2 \times 14$ and $2 \times 2 \times 14$ arrays in Figure $1(\mathrm{~B}, \mathrm{C})$. The plasmonic induced density of the $2 \times 2 \times 14$ array is given in Figure 1D.

As the number of chains in the array increases the plasmon blueshifts toward the visible range $(1.7 \mathrm{eV}-3.0 \mathrm{eV})$ as seen in Figure $1 \mathrm{~A}$ inset. The blueshift is in accordance with TD-DFT results for Au nanowires for which the plasmon energy increases with the nanowire diameter. ${ }^{24}$ However, between the planar arrays of three and four Au chains, the plasmon energy does not increase but slightly decreases. Thereafter, the plasmon energy increases again, although rather moderately and monotonically, up to the array of $8 \mathrm{Au}$ chains. 
A)

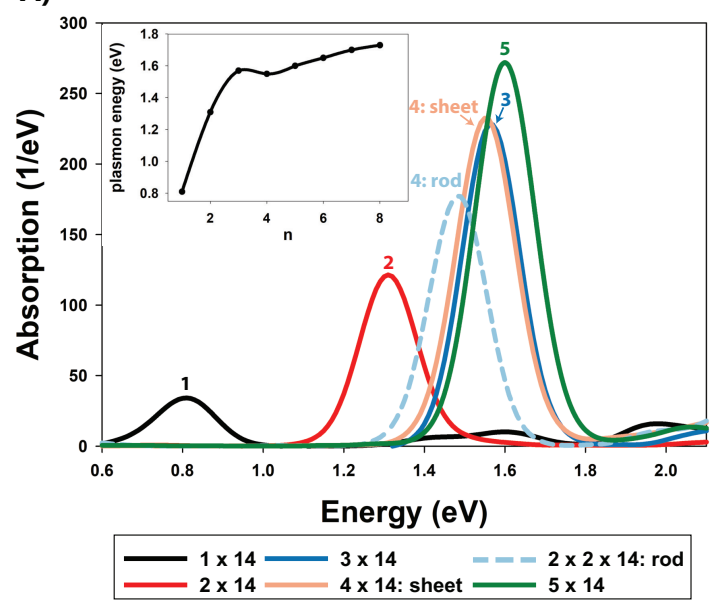

B)

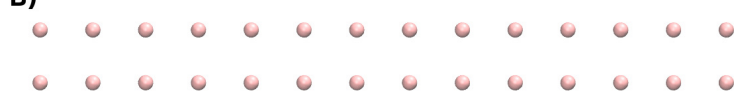

C)

88888888888888

88888888888888

Figure 1: A) Absorption spectra of Au arrays formed by one to five chains of $14 \mathrm{Au}$ atoms. The arrays of 2, 3, and 5 chains are planar. The 4 chain system is arranged into a 4 x 14 planar array (4: sheet) and a $2 \times 2 \times 14$ nanowire (4: rod). Inset - plasmon energy vs number of chains in an $n \times 14$ array. B and C) Atomic structures of the planar $2 \times 14$ array and $2 \times 2 \times 14$ nanowire. D) The total plasmonic transition density for the $2 \times 2 \times 14$ nanowire at $\omega=1.48 \mathrm{eV}$.

\section{Nodal structure and its effect on plasma frequency}

The non-monotonic behavior for the smallest arrays, which does not arise classically, is due to the quantum mechanical confinement of the average electron density between the $\mathrm{Au}$ chains and the evolution of the nodal structure of occupied state valence electron wavefunctions perpendicular to the Au chains. The sp-delocalized states in the finite array have transverse (with nodal surfaces parallel to the chain axis) and longitudinal nodes. The type and number of nodes determines the energetic ordering and occupancy of the states which produces a characteristic nodal structure of the finite system. The discussion is simplified by designating the $\mathrm{KS}$ orbitals as $(l, t)$, where $l$ is the number of longitudinal nodes and $t$ is the number of transverse nodes. Then the sp-delocalized states are classified into "subbands" corresponding to a group of sp-delocalized states having a certain number of transverse nodes. The first and second subbands, e.g., consist of all the KS orbitals in $(l, 0)$ and $(l, 1)$, respectively. The lowest energy orbital in each subband has zero longitudinal nodes.

The number of transverse nodes within the sp-delocalized states increases when chains are added to the array. Depending on the nodal structure and number of electrons, arrays of varying 
width have different partly filled or opened subbands. The opened subbands can participate in electronic transitions.

The subband structure of the arrays is illustrated as Fermi Quarter plots in Figure 2A. The nodal structure is extracted from the ground state with a two-dimensional sin transformation and the 6s-part of the gold wavefunctions. See Supporting Information for the computational details. The $\mathrm{x}$ and $\mathrm{y}$ coordinates of a Fermi Quarter plot correspond to the number of longitudinal and transverse nodes, respectively. The bottom left square represents the orbital with no longitudinal and transverse nodes, $(0,0)$, and each row of squares corresponds to a certain subband with different number of longitudinal nodes. Colored squares indicate the occupancy of a particular $(l, t) \mathrm{KS}$ orbital and the intensity is proportional to the magnitude of the two-dimensional sin transformation. In the Fermi Quarter plot of the 1 x 14 array, squares with zero transverse nodes and zero to six longitudinal nodes are colored indicating $\mathrm{KS}$ orbitals $(l, 0)$ for $l=0-6$ are occupied and the subband with zero transverse nodes is opened. Within each subband, the orbital energy increases with the number of longitudinal nodes. Fundamental transitions will occur to the right by one box. Higher harmonic transitions have larger steps, and even step sizes are parity-forbidden within the centrosymmetric array. Purely inter-subband transitions, generating transverse plasmons, are forbidden for excitations with the external electric field along the chain axis.

The Fermi Quarter plots are a representation of what can otherwise be obtained by visual inspection of the KS orbitals. Select Kohn-Sham (KS) orbitals of 3 x 14 Au are shown in Figure 2B as an illustration. For example, orbital 227 has no transverse and nine longitudinal nodes and, correspondingly, the square $(9,0)$ is dark red in the Fermi Quarter plot indicating occupation. Subbands with zero and one transverse node, i.e., $(l, 0)$ and $(l, 1)$, are opened in the 3 x 14 array. For example, orbitals 227 and 229 have no transverse nodes and orbitals 228 and 230, which is the HOMO, have one transverse node. The $(l, 2)$ subband with two transverse nodes begins with the LUMO (orbital 231), which has no longitudinal nodes. This subband is unoccupied and so only the first two rows are lit up in the Fermi Quarter plot of the 3 x 14 array. As chains are added to the array, more rows become colored as the subbands become occupied. The growing "Fermi 


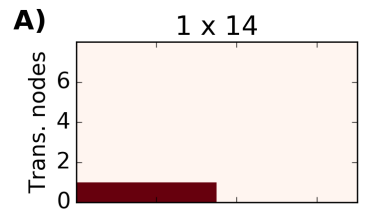

$2 \times 14$

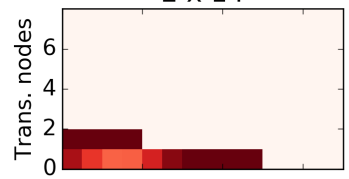

$3 \times 14$

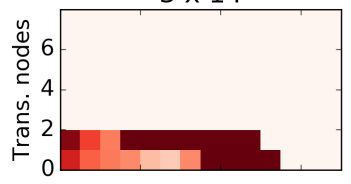

$4 \times 14$

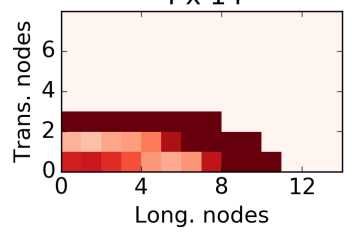

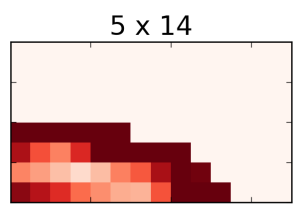

$6 \times 14$

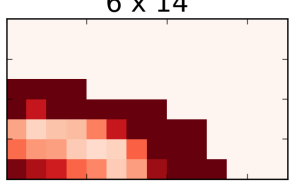

$7 \times 14$
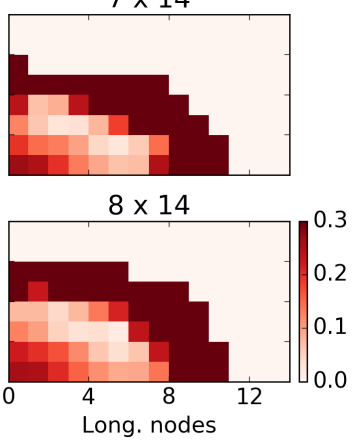

B)

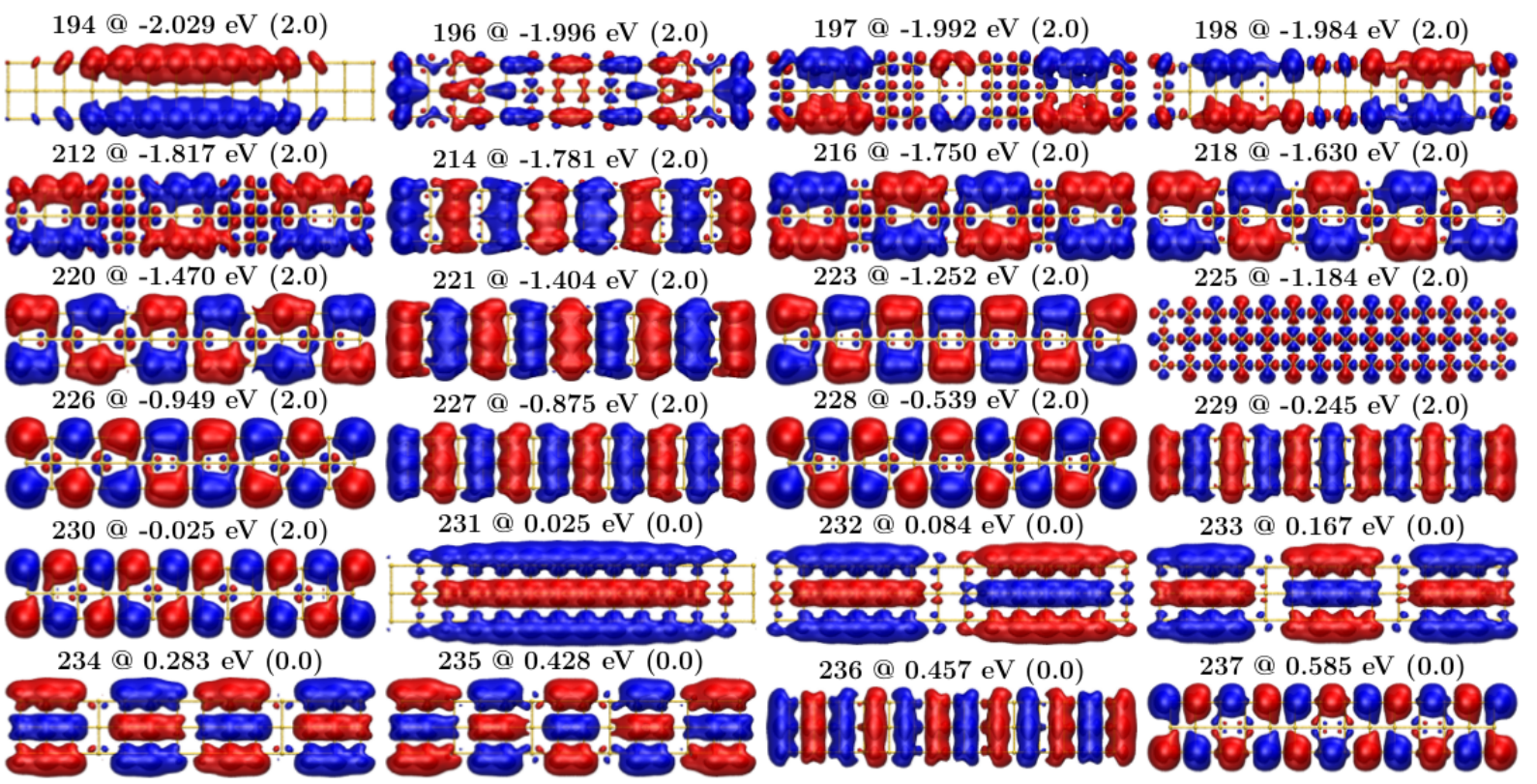

Figure 2: Subband structure in Au arrays. A) Fermi Quarter plot of Au arrays of different size. The intensity of a square is proportional to the $\mathrm{Au} 6 \mathrm{~s}$ character of the wave function obtained by a sin Fourier transform. B) Select KS orbitals of a $3 \times 14$ Au array showing the nodal structure and delocalization across the array. The label for each KS orbital corresponds to the orbital number, energy level with respect to the Fermi level, and occupancy in parentheses. 
circle" shows the emergence of the more bulk like electronic structure. A schematic of the highest order subband in the occupied states and lowest order subband in the unoccupied states in different arrays, i.e., $(0, t)$ and $(0, t+1)$, is given in Figure 3.

The orbitals with large Au 6s character are brightest in the Fermi Quarter plots. The more energetically stable $\mathrm{Au}$ sp-states hybridize with the d-orbitals and obscure the nodal structure. For example, orbitals 197 and 198 in the $3 \times 14$ array (Figure 2B) have one transverse node, but the localized d-states hinder identification of the longitudinal nodes. The magnitude of the sin transformation is diminished and the corresponding square in the Fermi Quarter plot is less intense. Orbital 225 has d-orbital character with weaker density at the array ends.
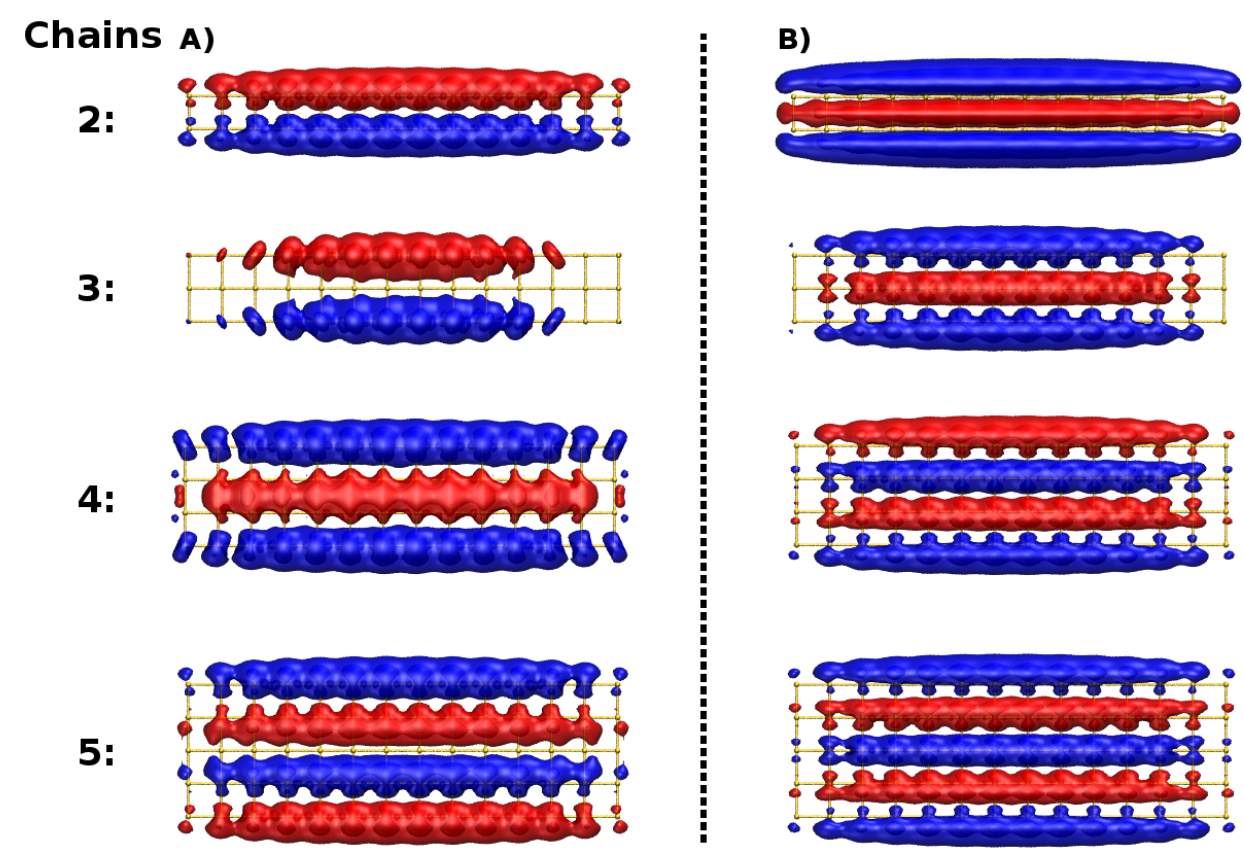

Figure 3: Schematic of the subband structure in Au arrays. A) Highest order subband in the occupied KS orbitals, i.e., within the open subbands. B) Lowest order subband in the unoccupied KS orbitals. The lowest energy states without longitudinal nodes, $(0, t)$ and $(0, t+1)$, are shown on each row.

The plasmonic redshift between the three and four chain Au arrays is, in part, related to the unique transversal nodal structure of the three chain array within the subbands with occupation. Unlike the other sizes, the single node in the three chain array is far from the array edges, i.e., on top of the middle Au chain as seen in Figure 3A, resulting in weak spillout. Conversely, the four 
chain array has two nodal planes between the chains nearest to the array edges where they cause the electron density to spillout from between the chains. The effect of the spillout is strong enough to decrease the plasmon frequency. The weak electron spillout of the triple chain array is also expressed in its relatively small work function and shown in the density of states with respect to the vacuum level in Figure S1. The variation of the spillout with the number of Au chains can be seen even more directly in the decays of the electron densities to the vacuum as depicted in Figure S2. Occupying more subbands in larger arrays does not have as pronounced of an influence and the change to the work function is minor. Thus, after the four chain array the plasmon frequency increases modestly and monotonically up to arrays of eight chains.

In a similar manner, the electron spillout from the $2 \times 2 \times 14$ nanowire is larger than that from the planar 4 x 14 array and the plasmon frequency redshifts from the array to the nanowire. The transverse nodes in the nanowire are between two $\mathrm{Au}$ chains on each face so that the plasmon frequency is even lower than that of the $3 \times 14$ array. However, the electron density corresponding to the lowest subband has a tendency to confine in the middle of four Au chains resulting in a plasmon frequency larger than that for the 2 x 14 array.

\section{TCM analysis of electronic excitations in the Au double chain}

A more detailed quantum mechanical understanding of the plasmonic absorption of Au arrays is gained by inspecting the KS electron-hole transition contributions visualized as TCMs. We pay particular focus to the $2 \times 14 \mathrm{Au}$ double chain because its characteristics will be compared with those of the simplest and most fundamental mixed arrays comprising only two chains of different atomic species. The TCM of the $2 \times 14 \mathrm{Au}$ double chain array for the molecular plasmon at $1.31 \mathrm{eV}$ is given in Figure 4A. The two regions of red illumination correspond to the two individual electron-hole transitions which form the plasmon. The energies of the initial and final states are on the horizontal and vertical axis of the TCM plot. The absorption energy is noted as $\omega$ and the line $\omega=\varepsilon_{u}-\varepsilon_{o}$ corresponds to the electron-hole excitations which are not affected by the Coulomb and exchange-correlation kernel interactions. For more details about the TCM see the 
Methods section. The brighter, i.e. stronger, contribution corresponds to the KS transition between delocalized sp-states with 9 and 10 longitudinal nodes and no transverse nodes, i.e., $(9,0)$ and $(10,0)$, as shown in the KS orbitals next to the DOS. The difference in the numbers of longitudinal nodes is one, which produces the dipolar total induced density in the inset.

The formation of bonding (no transverse nodes) and antibonding (one transverse node) states within the electronic structure of the double chain array produces the subband structure discussed above. The HOMO and LUMO, i.e., $(3,1)$ and $(4,1)$, are within the subband containing one transverse node, and the HOMO $\rightarrow$ LUMO transition is weakly illuminated, i.e. a weaker contribution, in the lower right corner of the TCM. The strongest induced dipole moment is, however, between bonding states.

Both the TCM of the strong absorption peak in the double chain array in Figure 4A and the TCM for the single chain in Figure S3 show one dominant electron-hole transition contribution. The HOMO $\rightarrow$ LUMO transition contributes most strongly to the molecular plasmon in a single Au chain, which is in accordance with previous predictions. ${ }^{24}$ In both, the plasmon resonance energy, $\omega$, is significantly larger than the KS eigenvalue difference of the corresponding transition, which is indicated by the dotted line. This shift is a result of the strong influence of the Coulomb interaction taken into account in TD-DFT, and obtained as a gradual evolution in the scaling method. ${ }^{25,27}$ Notably, the TCM visually illustrates the increase in the excitation energy due to the Coulomb interactions captured using other methods such as GPI. ${ }^{31}$ In this respect, these resonances can be attributed as molecular plasmon excitations despite being comprised of only one dominant transition.

\section{TCM analysis of electronic excitations in wide Au arrays}

The TCMs of the molecular plasmons in wider Au arrays are shown in Figure 5. The transverse nodes and subband structure of the larger arrays permit more sp-type KS states close to the Fermi level. Opening more subbands increases the number of electron-hole transitions contributing to the molecular plasmons of larger arrays. Both open subbands, $(l, 0)$ and $(l, 1)$, contribute to the 

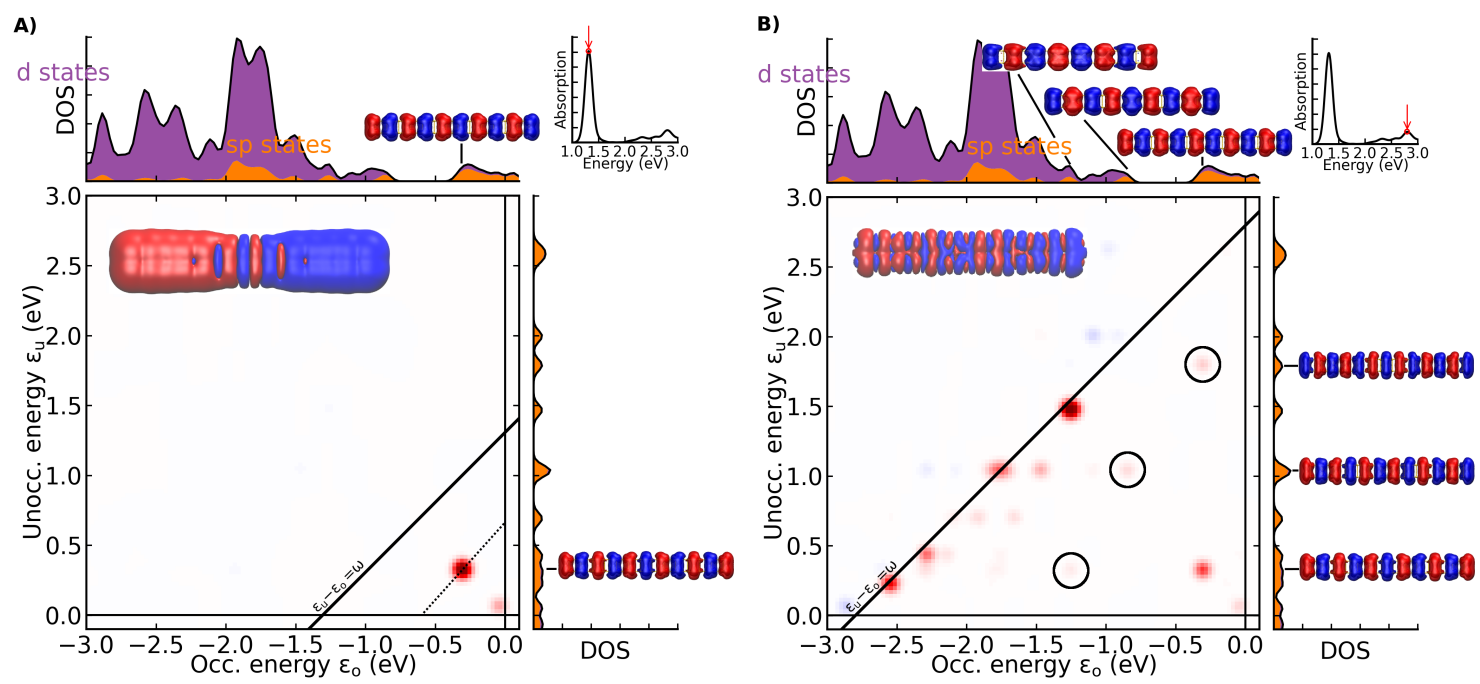

Figure 4: Transition Contribution Maps for the $2 \times 14 \mathrm{Au}$ array at A) the plasmonic peak at $\omega$ $=1.31 \mathrm{eV}$ and $\mathrm{B}$ ) at a higher energy peak with contribution from single-particle and collective electron transitions at $\omega=2.80 \mathrm{eV}$. The KS orbitals involved in the plasmonic transition are shown next to the DOS and the total transition densities are shown in the insets. For example in A) the dominant contribution is formed by the $(9,0) \rightarrow(10,0) \mathrm{KS}$ transition.

molecular plasmon in the $2 \times 14$ and the $3 \times 14$ arrays, and the contribution from the $(l, 1)$ subband intensifies in the $3 \times 14$ array, and their KS orbitals are provided next to the respective TCMs. The plasmon of the $4 \times 14$ planar array is formed by contributions from the $(l, 0),(l, 1)$ and $(l, 2)$ subbands. A similar increase in the contributions from opened subbands is observed in higher dimension nanorods. The TCM of the plasmon of the $2 \times 2 \times 14$ nanorod has strong contributions from the $(l, 0,0)$ and the degenerate $(l, 0,1)$ and $(l, 1,0)$ subbands, where the third value indicates the node perpendicular to the longitudinal and transverse nodes. In this manner, the collective nature of the molecular plasmon (in this instance collectivity refers to the number of contributing electron-hole transitions) swells with the array size producing a strong dipolar induced density.

An alternative method of quantifying the number of individual KS transitions to the absorption is by means of the Inverse Participation Ratio (IPR). ${ }^{8}$ A singular electron-hole excitation has an IPR value equal to 1 , and the growing collectivity of a photoabsorption mode is expressed as increasingly large values. IPR does not, however, discern whether these excitations have been 
affected by the Coulombic interaction. It is calculated as

$$
\mathrm{IPR}=\frac{\left(\sum_{I} s_{I}^{2}\right)^{2}}{\sum_{I} s_{I}^{4}}
$$

where $s_{I}$ are the singular values of the matrix consisting of the weighted contributions to the photoabsorption spectrum (see Eq. (17) of Ref.), ${ }^{38}$ which is also visualized in the TCM. The IPRs of the single and double chain arrays are both 1.0 representing the single dominant transition in the TCMs. The IPR increases to 1.6 in the triple chain array and 2.7 in the array with four chains. The intense absorption peak of an array of eight chains has an IPR equal to 4.5. The increase of this index with the array size reflects the growing amount of transitions from the increased number of occupied subbands contributing to the absorption, and further demonstrates that the strong low-energy absorption peak is not a single-particle-like transition. These trends are also visible in the TCM, which also provides more information such as the energy, magnitude, and sign of the contributions.
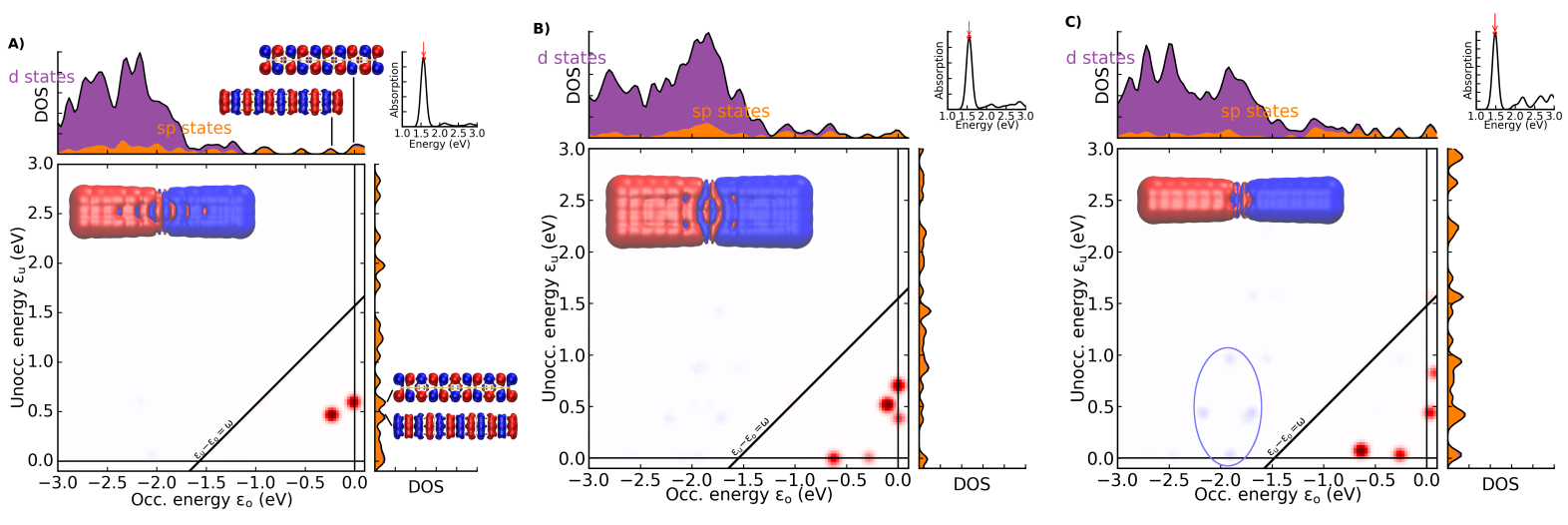

Figure 5: Transition Contribution Maps A) for the $3 \times 14 \mathrm{Au}$ array at the plasmonic peak at $\omega=$ $1.57 \mathrm{eV}, \mathrm{B}$ ) for the planar $4 \times 14 \mathrm{Au}$ array at the plasmonic peak at $\omega=1.55 \mathrm{eV}$, and C) for the rod-like $2 \times 2 \times 14 \mathrm{Au}$ nanowire at the plasmonic peak at $\omega=1.48 \mathrm{eV}$. Total transition densities are shown in the insets.

We also note that the d-levels of the triple chain array are lower in energy with respect to the Fermi level than those of the double chain array. This stability reflects the more compact electronic structure of the former system discussed earlier. In both the sheet-like and rod-like arrays of four $\mathrm{Au}$ chains the stronger splitting between bonding and antibonding d-states pushes the d-levels 


\section{TCM analysis of electronic excitations above the plasmon energy}

In the above TCMs for molecular plasmonic excitations, the main contributing KS transitions appear as red spots below the probe line corresponding to the plasmon resonance energy, $\omega=\varepsilon_{u}-\varepsilon_{o}$. These transitions remain weakly illuminated when probing higher energy resonances. We present the TCM of the double chain Au array at an excitation peak of $2.80 \mathrm{eV}$ as an example in Figure 4B. Higher harmonic molecular plasmons also contribute to this absorption peak and are circled. In these electron-hole transitions the difference in the number of longitudinal nodes is three. Similar higher order molecular plasmons have been identified in atomic chains by Bernadotte et al. using the $\lambda$-scaling method ${ }^{25}$ and in the edges of $\mathrm{MoS}_{2}$ nanoflakes. ${ }^{13}$ The TCM clearly shows that the photoabsorption peak has contributions from both the first and higher order plasmons below the probe line and single-particle excitations on the probe line. The induced density in the inset of the figure is a product of the mixing and constructive interference between the dipole moments of each contribution. This mixing of plasmonic and single-particle transitions offers a possibility of generating hot electrons at the dense d-states. There are rather few transitions below the line weakly contributing to non-specific d-states, and so hot electron generation is not expected to be an intense process in double chains. Arrays of several atomic chains would be more effective. 


\section{Molecular plasmons in mixed arrays}

In this sub-section, we focus on mixed, or coupled, arrays comprising two chains each fourteen atoms long. By mixing homonuclear chains $(\mathrm{Au}, \mathrm{Ag}, \mathrm{Cu}, \mathrm{Pd}, \mathrm{Pt})$, the position of the surface plasmon resonance can be tuned or the molecular plasmon fragmented into several peaks with broadband photoabsorption. For example, as seen in Figures 6(A,D) the single plasmon peak of $14 \mathrm{Cu}-14 \mathrm{Ag}$ double chain is situated halfway between the plasmon peak of pure $\mathrm{Ag}$ and pure $\mathrm{Cu}$ double chains. However, when the $\mathrm{Cu}$ chain is joined with the Au chain, the plasmon peak is shifted toward that of the pure Au double chain (Figures 6(B,E)), and when the Ag chain is joined with the Au chain, the plasmon peak is practically at that of the pure Au double chain (Figures 6(C,F)). In striking contrast, when a $\mathrm{Au}$ chain is joined with a $\mathrm{TM}(\mathrm{Pd}, \mathrm{Pt})$ chain, the plasmon modes are highly suppressed and several peaks form as seen in the case of the 14Pd-14Au double chain in Figure 7.

A)
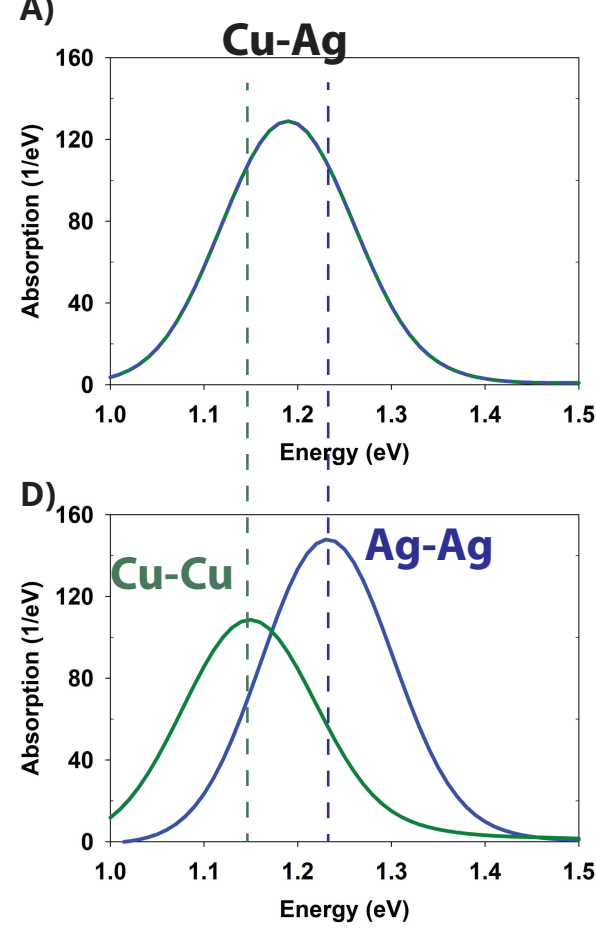

B)

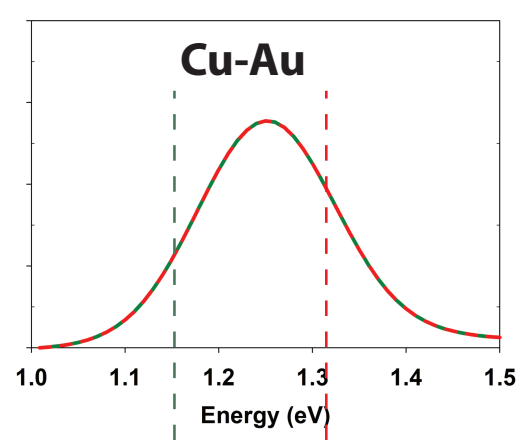

E)

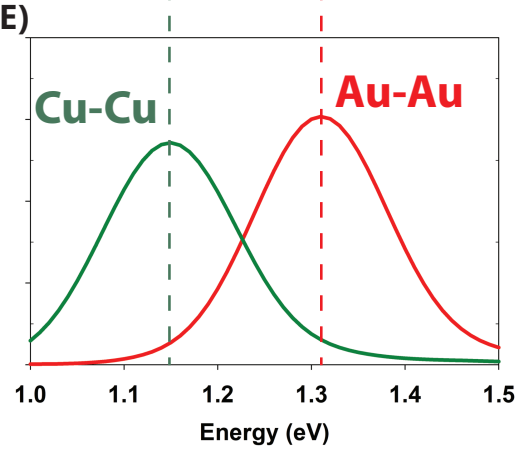

C)

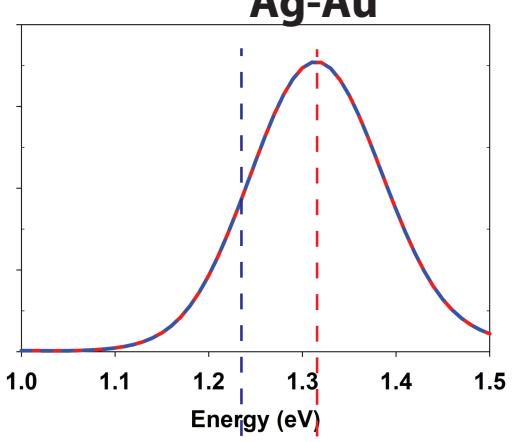

F)

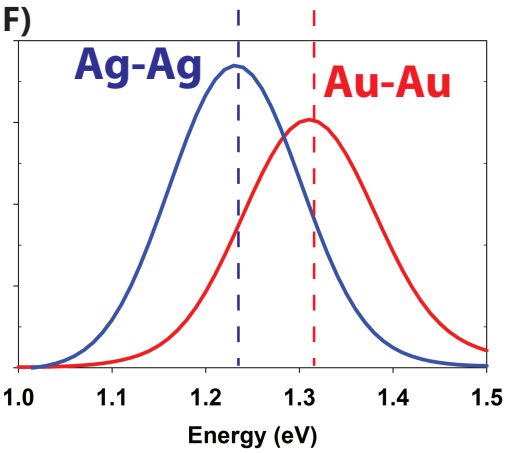

Figure 6: Photoabsorption spectra for mixed arrays comprising two chains of 14 atoms of A) $\mathrm{Cu}$ $\mathrm{Ag}$ (maximum at $\omega=1.19 \mathrm{eV}$ ), B) $\mathrm{Cu}-\mathrm{Au}(1.25 \mathrm{eV}$ ), and $\mathrm{C}$ ) $\mathrm{Ag}-\mathrm{Au}(1.31 \mathrm{eV}$ ) double chains. D-F) Spectra for the pure $\mathrm{Cu}-\mathrm{Cu}(1.15 \mathrm{eV}), \mathrm{Ag}-\mathrm{Ag}(1.23 \mathrm{eV})$, and $\mathrm{Au}-\mathrm{Au}(1.31 \mathrm{eV}) 2 \mathrm{x} 14$ double chains. 


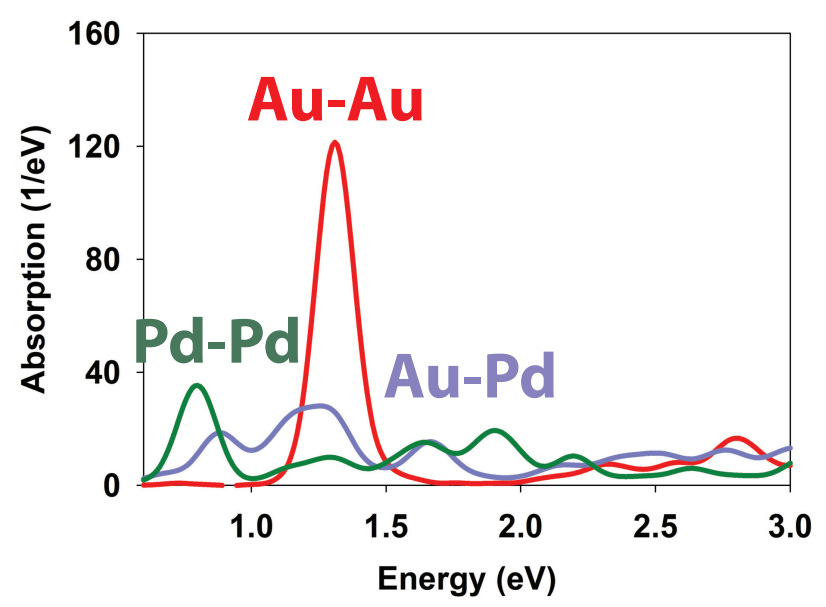

Figure 7: Photoabsorption spectra of the mixed double chain array comprising one chain of $14 \mathrm{Pd}$ and one chain of $14 \mathrm{Au}$ atoms, and pure $\mathrm{Pd}-\mathrm{Pd}$ and $\mathrm{Au}-\mathrm{Au} 2$ x 14 double chains.

The behavior of the molecular plasmon in the double chains comprised of two different noble metals, which are shown in Figure 6, are first explained by the electron confinement depending on the atomic ionization potentials and atomic size. Thereafter the trends are further analyzed using TCMs. The photoabsorption spectrum of the 14Pd-14Au double chain more prominently reflects the chemical properties of the two species and requires a quantum mechanical analysis at the outset.

\section{Analysis of electronic excitations in mixed noble metal arrays}

The shift of plasmon frequency in the mixed arrays of homonuclear chains is explained using the change of the total electron density from the constituent pure arrays. The imbalance of the electron density within the mixed arrays is described in the analysis below using the differences in the ionization potentials ${ }^{45}$ and valence electron density decay lengths ${ }^{46}$ between the constituent species.

The plasmon frequency for the mixed $\mathrm{Cu}-\mathrm{Ag}$ double chain array is located at the average of the plasmon frequency of the $\mathrm{Cu}-\mathrm{Cu}$ and $\mathrm{Ag}-\mathrm{Ag}$ double chain arrays as seen in Figure 6(A,D). The electron density shifts toward the chain with the higher ionization potential, and thereby electron affinity, which in this case is $\mathrm{Cu}$. Both adjacent to the array and within the atomic lattice, the 
electron density around the $\mathrm{Cu}$ chain increases while decreasing around the Ag chain. The difference in the ionization potentials is small and so the corresponding shift of the electron density is relatively small. The dipole moment of the $\mathrm{Cu}-\mathrm{Ag}$ mixed array is $-1.2|e| \AA$ transverse to the chain axis. The electron density of the $\mathrm{Cu}-\mathrm{Ag}$ mixed array is approximately equal to the average of the two pure arrays as seen in Figure S4. The shifting was validated by explicitly calculating the total electron density as described in the Supporting Information.

In the $\mathrm{Cu}-\mathrm{Au}$ double chain array, the plasmon frequency is also between the two pure arrays, but shifted slightly more toward the frequency of the pure Au array as seen in Figure 6(B,E). The ionization potential of $\mathrm{Au}$ is greater than that of $\mathrm{Cu}$, and the $\mathrm{Au}$ chain draws the electron density away from the neighboring chain reducing the electron spillout from the $\mathrm{Cu}$ chain (Figure S4). Due to the large ionization potential difference the magnitude of the shift of total electron density is greater than in the $\mathrm{Cu}-\mathrm{Ag}$ array, and the dipole moment of the $\mathrm{Cu}-\mathrm{Au}$ mixed array is $2.4|e| \AA$. The shift is limited by the fixed interchain distance and small electron density decay length of the $\mathrm{Cu}$ chain relative to $\mathrm{Ag}$. Therefore the resulting electron density around the $\mathrm{Cu}$ and $\mathrm{Au}$ chains does not rise very much from the average of the $\mathrm{Cu}$ and $\mathrm{Au}$ pure arrays, and the plasmon frequency is shifted modestly from the midpoint. The explicit calculation of the total electron density as described in the Supporting Information validates the shifting of the electron density.

Finally, the mixed Ag-Au double chain array is composed of chains with similar electron density decay lengths and a large ionization potential difference. The shift of the electron density toward the Au chain is the largest of the mixed arrays considered and its dipole moment is $3.4|e|$ $\AA$. Similar to the $\mathrm{Cu}$-Au double chain array, there is decreased spillout at the chain with the smaller electron affinity as seen in Figure S4. Moreover because the difference between the plasmon resonance energy of the pure $\mathrm{Ag}$ and Au double chain arrays is relatively small, the plasmon frequency of the mixed Ag-Au array ends up being nearly the same as that of the Au-Au array. The shifting is is validated by explicit calculation of the total electron density as described in the Supporting Information.

We will now turn to the more detailed description based on TCMs which are shown in Figure 8 
for $\mathrm{Cu}-\mathrm{Cu}, \mathrm{Cu}-\mathrm{Au}$, and $\mathrm{Au}-\mathrm{Au}$ double chain arrays. In each case, the plasmon excitation has one dominant KS transition between delocalized sp-states and the strong dipolar induced density is similar to the pure Au double chains. The shifted electron density of the mixed array produces a transition density which is also asymmetric with respect to the plane between the chains. The asymmetric transition density is shown in the TCM inset in Figure 8B. In this figure series, the shift of the plasmon energy is related to the energy of its constituent dominating KS transition. For example in a mixed $\mathrm{Cu}-\mathrm{Au}$ array, seen in Figure 8, the occupied state of the collective excitation is shifted midway between that of a pure $\mathrm{Au}$ and and pure $\mathrm{Cu}$ array. The energy of the initially occupied $(9,0)$ state of the KS transition is related to the ordering of the orbitals within the nodal structure and select KS orbitals are given in Figure S5. In the pure Au array the $(9,0)$ state is energetically more stable than the entire $(l, 1)$ subband. Whereas in the pure $\mathrm{Cu}$ array, having a node between the smaller $\mathrm{Cu}$ atoms is more stable and the $(9,0)$ state rises above the $(2,1)$ orbital. The KS orbitals of the array can be thought of as a construction of the KS orbitals of two individual chains which form a bonding and an anti-bonding orbital. In the mixed array, the energy mismatch between the KS orbitals of $\mathrm{Au}$ and $\mathrm{Cu}$ chains produces a bonding KS orbital energetically between the KS orbitals of the pure $\mathrm{Cu}$ and pure $\mathrm{Au}$ arrays. The unoccupied states, formed by a bonding KS orbital with an additional node, remain at approximately the same energy, and the plasmon resonance energy of the mixed array follows the single particle excitation energy. The plasmon formed in the $\mathrm{Ag}-\mathrm{Au}$ and $\mathrm{Cu}-\mathrm{Ag}$ mixed double chains are constructed in a similar manner to the $\mathrm{Cu}$-Au mixed arrays and the TCMs are shown in Figures S6 and S7. Each TCM has one dominant KS transition between the delocalized sp-states with 9 and 10 nodes and a weaker contribution from the $\mathrm{HOMO} \rightarrow$ LUMO transition.

\section{Analysis of electronic excitations in TM and in mixed noble metal-TM arrays}

Figure 7 shows a rather intense absorption peak at $0.80 \mathrm{eV}$ for the $2 \times 14 \mathrm{Pd}$ double chain. The TCM for this excitation in Figure 9A shows a structure with plasmonic characteristics, i.e., all KS transitions contributing strongly to the excitation are well below the $\omega$ probe line. The region just 

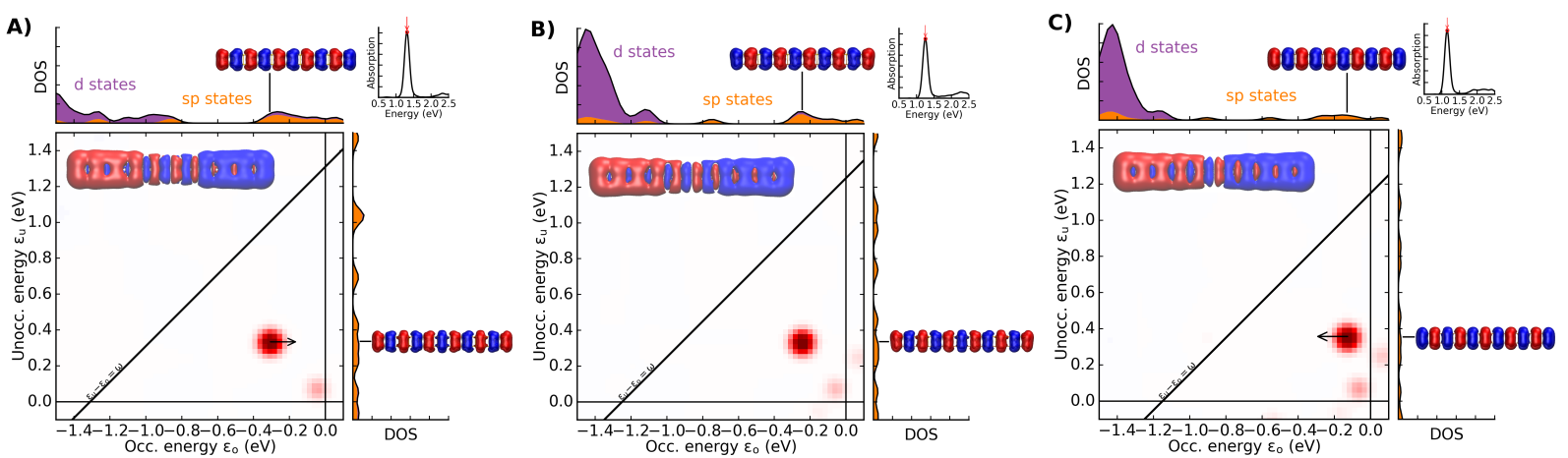

Figure 8: Transition contribution maps for plasmonic excitations in $\mathrm{A}$ ) $\mathrm{Au}-\mathrm{Au}, \mathrm{B}) \mathrm{Cu}-\mathrm{Au}$, and $\mathrm{C}$ ) $\mathrm{Cu}-\mathrm{Cu} 2 \times 14$ double chains at $\omega=1.31,1.25$, and $1.15 \mathrm{eV}$, respectively. The arrow indicates the direction the $(9,0) \rightarrow(10,0) \mathrm{KS}$ transition moves when the array is mixed.

inside the lower right corner of the TCM and marked by the red spot contributes to the collective excitation. This region consists of two nearly degenerate transitions between the KS orbitals shown adjacent to the DOS. The localized d-states lessen the dipole moment of the transitions and dampen the intensity of the mode. In spite of the high energy d-levels, the array is able to sustain such a plasmonic excitation, but this may not be the case in other array sizes.

The photoabsorption spectrum of the Pd-Au double chain in Figure 7 is significantly suppressed and fragmented compared to the pure and mixed noble metal double chains. The fragmentation is due to both sd-hybridization and transient d-transitions between the overwhelming amount of occupied d-states near the Fermi level introduced by Pd and shown in the DOS in Figures 9(BD). While the fleeting transitions between d-states contribute to the photoabsorption only near the probe line, some transitions continue to contribute at higher energies. These transitions occur between sd-hybridized states. TCMs conveniently show that the contributions of these individual transitions between sd-hybridized states vary and even change sign near the $\omega$-probe line. For example, we consider the two KS transitions labelled A and B in Figure 9B. The initially occupied state of transitions A and B each have eight nodes on the Au chain, but they have hybridized with the either the delocalized $\mathrm{d}_{y z}$ (in $\mathrm{A}$ ) or $\mathrm{d}_{z}^{2}$ (in B) states on the Pd chain. The different symmetry on the Pd chain produces partial induced densities where the Au chain is either in phase with the Pd chain (transition A) or out of phase (transition B) as seen in Figure 9E. The final state of transitions 

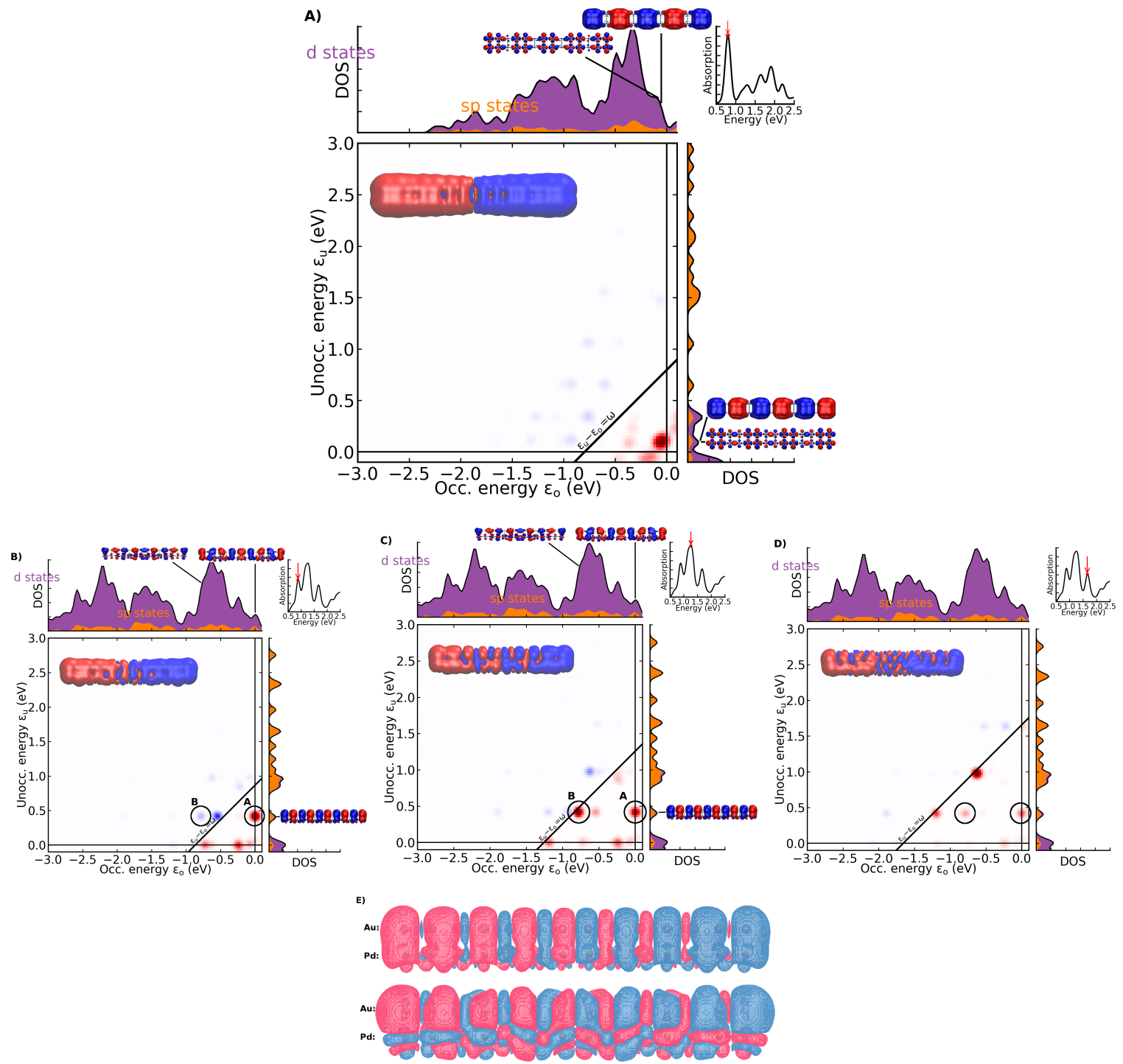

Figure 9: Plasmons from pure Pd-Pd and mixed Pd-Au arrays. A) Contribution to the plasmonic excitation at $\omega=0.80 \mathrm{eV}$ of a Pd-Pd array. B-D) Contribution to the absorption peak of a Pd-Au mixed array at $\omega=0.87,1.26$, and $1.66 \mathrm{eV}$, respectively. E) Isolated transition densities of the transitions A (top) and B (bottom) which are formed from the KS orbitals shown next to the DOS in (C). 
A and $\mathrm{B}$ has nine nodes, and due to the longitudinal nodal difference of one, both partial induced densities have an overall dipole. There is pronounced screening from the localized d-states in both partial induced densities. The KS orbitals of transitions A and B are given in Figure 9(B,C). In Figure 9C transitions $\mathrm{A}$ and $\mathrm{B}$ contribute positively to the absorption peak at $\omega=1.26 \mathrm{eV}$. However, in Figure 9B for the absorption peak at $\omega=0.87 \mathrm{eV}$ transition $\mathrm{B}$ and the adjacent d-transition have changed sign from positive to negative. Other resonances, such as those at $\omega=1.16$ or $1.66 \mathrm{eV}$ as seen in Figure 9D, are produced by changes of the contribution in other active KS transitions. The numerous closely spaced transitions effectively broaden the absorption band. In this manner, the sd-hybridization fragments the plasmon by activating additional KS transitions leading to the additional peaks in the photoabsorption spectra. ${ }^{38,47}$ As the resonance energy reduces, the total induced density becomes more dipolar as a result of the constructive interference of the dipole moments (see Figure 9(B-D) insets).

Similar effects are observed in other Au-TM mixed arrays (Pt, Ni, Fe) with d-levels near the Fermi level. ${ }^{48}$ The spectra and TCMs of pure Pt double chain arrays and 14Au-14Pt mixed arrays are shown in Figures S8 and S9, respectively.

\section{Conclusions}

We have analyzed the optical properties of multiple pure and mixed noble/transition metal planar nanoarrays of fixed length and varying width. A strong absorption mode blueshifts toward the visible range when the width of the array increases, but the developing subband structure, due to the quantum confinement in the finite atomic arrays, produces a non-monotonic shift. These effects are seen when the electron spillout and the work function increase from the three Au chain to the four Au chain causing a redshift in the absorption.

The strong absorption mode is produced by transitions between sp-delocalized states that are low in energy in comparison to the resonance frequency indicating the molecular-plasmonic character of the mode. Transition Contribution Maps provide insightful visualization of these aspects. 
The response of a single Au chain exhibits the same features. As the width of the nanoarray is increased, transitions originating from the newly opened subbands become more numerous. The contribution of several transitions and the developing nodal structure in atomic arrays are instructive in understanding the behavior of larger nanoparticles. Although the nodal structure is more complex in larger nanoparticles, it is evident that the plasmons of larger nanoparticles have contributions from more KS transitions, some of which may fragment the plasmon excitation. ${ }^{38,47}$

The energy position of the d-electron levels affects the optical properties of mixed arrays of homonuclear chains. The intense plasmon is either maintained when mixing noble metal chains, or fragmented when mixing a noble metal chain with a TM chain. The sd-hybridization and introduced d-states activate additional KS transitions to produce extra resonances. These resonances fragment and diminish the photoabsorption intensity. We have mainly focused on Au chains mixed with Pd or Pt atoms, but as pure $\mathrm{Ag}$ and $\mathrm{Cu}$ systems behave similarly to pure Au system, one can expect a similar picture when $\mathrm{Ag}$ and $\mathrm{Cu}$ are doped with $\mathrm{TM}$ atoms.

The free-standing model we have employed allows the contributions from the discrete electronhole transitions to be clearly identified and analyzed. The methodology described here not only improves the understanding of collectivity and plasmonicity of the strong absorption modes in small systems, but may be applicable to different optical technologies provided that such multichain systems are built experimentally on a "neutral" substrate. Further this work could improve the description of the electronic transitions within higher dimensional or chemically heterogeneous systems.

\section{Methods}

Atomic wires comprising noble $(\mathrm{Au}, \mathrm{Ag}, \mathrm{Cu})$ or transition $(\mathrm{Pd}, \mathrm{Pt})$ metal atoms with length varying from 2 to 14 atoms are constructed. Up to eight chains are assembled to form pure or mixed arrays of homonuclear chains (see Figure 1B for a homonuclear array). To mimic supported nanostructures and to allow a clear identification and analysis of the discrete electron-hole transitions, a 
square (simple cubic) lattice is assumed for planar (rod-like) arrays and the bond length is set to $2.89 \AA$, as measured in experimentally realized Au chains on the $\mathrm{NiAl}(110)$ substrate. ${ }^{14}$

The electronic structures of the nanostructures are calculated within DFT ${ }^{49,50}$ using the solidstate modified GLLB-SC exchange-correlation potential ${ }^{43}$ which accurately describes the energy level positions of the d-states in noble metals. ${ }^{51,52}$ We employ the GPAW code package ${ }^{44,53-55}$ based on the projector-augmented wave (PAW) method $^{56}$ and utilize the ASE package. ${ }^{57}$ Electronic wavefunctions are expanded as linear combinations of atomic orbitals (LCAO) ${ }^{58}$ with less than $0.05 \mathrm{eV}$ Fermi-Dirac smearing of the occupation numbers. The pseudo wavefunctions are evaluated in real space with a grid spacing of $0.3 \AA$ and the electron density and potentials are evaluated on a doubly fine $0.15 \AA$ grid. The molecules are surrounded by at least $6 \AA$ of vacuum and the Hartree potential is evaluated on a larger and coarser grid with at least $48 \AA$ of vacuum.

The optical response of the atomic wires and arrays is calculated using the LCAO time propagation (TP) TD-DFT code ${ }^{44}$ with weak $\delta$-pulse perturbation. ${ }^{39}$ In this approach, the electron wavefunctions are evolved after an external electric (in dipole approximation) $\delta$-pulse along the long wire axis. The time-dependent induced density provides the time-dependent dipole moment from which the dynamical polarizability and the photoabsorption spectrum over all frequencies are determined. The photoabsorption spectrum with Gaussian broadening of $\sigma=0.07 \mathrm{eV}$ is sufficiently obtained using a time step of 10 as for a total propagation time of $30 \mathrm{fs}$. Some of the nanowire systems were also calculated using Gaussian $03^{59}$ with a B3PW91 hybrid functional ${ }^{60-62}$ and a LanL2DZ basis set. ${ }^{63}$ The main features of the absorption spectra were consistent with the LCAOTP-TD-DFT calculations as described in the Supporting Information. ${ }^{48}$ With the exception of the photoabsorption spectra calculated using Gaussian 03 shown in the Supporting Information, all the results presented and analyzed in this paper are obtained with the LCAO-TP-TD-DFT method.

The induced density matrix between occupied and unoccupied KS states and the corresponding dipole matrix elements allows the photoabsorption spectrum to be decomposed into contributions from individual discrete electron-hole transitions with well-defined relative weights. ${ }^{38}$ The decomposition is represented as a two-dimensional Transition Composition Map with the initially 
unoccupied and occupied energies, $\varepsilon_{u}$ and $\varepsilon_{o}$, given on the vertical and horizontal axes of TCM, respectively. The density of states (DOS) along the top and side is colored to indicate the sp and $\mathrm{d}$ characters of the KS states. The different discrete electron-hole transitions are denoted by spots on the TCM plane with an intensity proportional to the normalized KS decomposition weight of the electron-hole transition $\left(S_{i a} / S\right)$ (see Eq. (17) and (21) of Ref.) ${ }^{38}$ The red (blue) color of the spots corresponds to the positive (negative) sign of the photoabsorption decomposition of the transition. The extent is given by the two-dimensional Gaussian broadening function $\left(\sigma_{\mathrm{TCM}}=0.04 \mathrm{eV}\right)$ and is distinct from the broadening of the photoabsorption spectrum, $\sigma$. The absorption energy is noted as $\omega$ and the line $\omega=\varepsilon_{u}-\varepsilon_{o}$ corresponds to the electron-hole excitations which are not affected by the Coulomb and exchange-correlation kernel interactions.

Similar to the photoabsorption decomposition into different electron-hole contributions, the induced electron density can be decomposed into partial densities corresponding to the different electron-hole transition contributions (see Eq. (18) of Ref.) ${ }^{38}$ Further details about these analysis tools can be found in Ref. ${ }^{38}$

\section{Acknowledgement}

The work was supported in part by US DOE Grant DE-FG02-07ER46354 (NN, VT, and TSR) and as part of the Academy of Finland Centre of Excellence program (project 251748, KMC, TPR, and MJP and project 312298, KMC). TPR also acknowledges support from the Knut and Alice Wallenberg Foundation and the Swedish Research Council. MK acknowledges funding from Academy of Finland under grant Nr. 295602. We acknowledge computational resources provided by CSC - IT Center for Science (Finland) and by the Aalto Science-IT project (Aalto University School of Science).

\section{Supporting Information Available}

We provide additional information on the Fermi Quarter plots, spillout of the electron density, pho- 
toabsorption decomposition for mixed arrays, and photoabsorption spectra obtained using Gaussian 03. This material is available free of charge via the Internet at http://pubs.acs.org.

\section{References}

1. Quinten, M.; Leitner, A.; Krenn, J. R.; Aussenegg, F. Electromagnetic Energy Transport via Linear Chains of Silver Nanoparticles. Opt. Lett. 1998, 23, 1331-1333.

2. Solis Jr., D.; Willingham, B.; Nauert, S. L.; Slaughter, L. S.; Olson, J.; Swanglap, P.; Paul, A.; Chang, W.-S.; Link, S. Electromagnetic Energy Transport in Nanoparticle Chains via Dark Plasmon Modes. Nano Lett. 2012, 12, 1349-1353.

3. Cortie, M. B.; McDonagh, A. M. Synthesis and Optical Properties of Hybrid and Alloy Plasmonic Nanoparticles. Chem. Rev. (Washington, DC, U. S.) 2011, 111, 3713-3735.

4. Rycenga, M.; Cobley, C. M.; Zeng, J.; Li, W.; Moran, C. H.; Zhang, Q.; Qin, D.; Xia, Y. Controlling the Synthesis and Assembly of Silver Nanostructures for Plasmonic Applications. Chem. Rev. (Washington, DC, U. S.) 2011, 111, 3669-3712.

5. Runge, E.; Gross, E. K. U. Density-Functional Theory for Time-Dependent Systems. Phys. Rev. Lett. 1984, 52, 997-1000.

6. Kümmel, S.; Andrae, K.; Reinhard, P.-G. Collectivity in the Optical Response of Small Metal Clusters. Appl. Phys. B: Lasers Opt. 2001, 73, 293-297.

7. Song, P.; Nordlander, P.; Gao, S. Quantum Mechanical Study of the Coupling of Plasmon Excitations to Atomic-Scale Electron Transport. J. Chem. Phys. 2011, 134, 074701.

8. Yasuike, T.; Nobusada, K.; Hayashi, M. Collectivity of Plasmonic Excitations in Small Sodium Clusters with Ring and Linear Structures. Phys. Rev. A 2011, 83, 013201.

9. Yin, H.-F.; Zhang, H. Quantum Mechanical Study on Plasmon Resonances in Small Ring Clusters. Int. J. Quantum Chem. 2012, 112, 2816-2821. 
10. Ma, H.; Gao, F.; Liang, W. Plasmon Resonance of Isolated Gold Hollow Nanoparticles and Nanoparticle Pairs: Insights from Electronic Structure Calculations. J. Phys. Chem. C 2012, $116,1755-1763$.

11. Yin, H.-F.; Zhang, H. Collectivity of Plasmon Excitations in Small Sodium Clusters with Planar Structure. Phys. B (Amsterdam, Neth.) 2012, 407, 416-420.

12. Wang, B.-J.; Xu, Y.; Ke, S.-H. Plasmon Excitations in Sodium Atomic Planes: A TimeDependent Density Functional Theory Study. J. Chem. Phys. 2012, 137, 054101.

13. Rossi, T. P.; Winther, K. T.; Jacobsen, K. W.; Nieminen, R. M.; Puska, M. J.; Thygesen, K. S. Effect of Edge Plasmons on the Optical Properties of $\mathrm{MoS}_{2}$ Monolayer Flakes. Phys. Rev. B 2017, 96, 155407.

14. Nilius, N.; Wallis, T.; Ho, W. Development of One-Dimensional Band Structure in Artificial Gold Chains. Science 2002, 297, 1853-1856.

15. Yan, J.; Yuan, Z.; Gao, S. End and Central Plasmon Resonances in Linear Atomic Chains. Phys. Rev. Lett. 2007, 98, 216602.

16. Yan, J.; Gao, S. Plasmon Resonances in Linear Atomic Chains: Free-Electron Behavior and Anisotropic Screening of $d$ Electrons. Phys. Rev. B 2008, 78, 235413.

17. Lian, K.-Y.; Sałek, P.; Jin, M.; Ding, D. Density-Functional Studies of Plasmons in Small Metal Clusters. J. Chem. Phys. 2009, 130, 174701.

18. Guidez, E. B.; Aikens, C. M. Quantum Mechanical Origin of the Plasmon: From Molecular Systems to Nanoparticles. Nanoscale 2014, 6, 11512-11527.

19. Nayyar, N.; Turkowski, V.; Rahman, T. S. Optical Generation of Collective Plasmon Modes in Small Gold Chains Induced by Doping Transition-Metal Impurities. Phys. Rev. Lett. 2012, 109, 157404. 
20. Freericks, J. K.; Turkowski, V. M.; Zlatić, V. F-electron Spectral Function of the FalicovKimball Model in Infinite Dimensions: The Half-Filled Case. Phys. Rev. B 2005, 71, 115111.

21. Nilius, N.; Wallis, T. M.; Ho, W. Tailoring Electronic Properties of Atomic Chains Assembled by STM. Appl. Phys. A: Mater. Sci. Process. 2005, 80, 951-956.

22. Puska, M. J.; Nieminen, R. M.; Manninen, M. Electronic Polarizability of Small Metal Spheres. Phys. Rev. B 1985, 31, 3486-3495.

23. Yannouleas, C.; Broglia, R. A. Collective and Single-Particle Aspects in the Optical Response of Metal Microclusters. Phys. Rev. A: At., Mol., Opt. Phys. 1991, 44, 5793-5802.

24. Piccini, G.; Havenith, R. W. A.; Broer, R.; Stener, M. Gold Nanowires: A Time-Dependent Density Functional Assessment of Plasmonic Behavior. J. Phys. Chem. C 2013, 117, 1719617204.

25. Bernadotte, S.; Evers, F.; Jacob, C. R. Plasmons In Molecules. J. Phys. Chem. C 2013, 117, $1863-1878$.

26. de Abajo, F. J. G.; Sapienza, R.; Noginov, M.; Benz, F.; Baumberg, J.; Maier, S.; Graham, D.; Aizpurua, J.; Ebbesen, T.; Pinchuk, A.; Khurgin, J.; Matczyszyn, K.; Hugall, J. T.; van Hulst, N.; Dawson, P.; Roberts, C.; Nielsen, M.; Bursi, L.; Flatté, M.; Yi, J. et al. Plasmonic and New Plasmonic Materials: General Discussion. Faraday Discuss. 2015, 178, 123-149.

27. Krauter, C. M.; Bernadotte, S.; Jacob, C. R.; Pernpointner, M.; Dreuw, A. Identification of Plasmons in Molecules with Scaled ab initio Approaches. J. Phys. Chem. C 2015, 119, $24564-$ 24573.

28. Ma, J.; Wang, Z.; Wang, L.-W. Interplay Between Plasmon and Single-Particle Excitations in a Metal Nanocluster. Nat. Commun. 2015, 6, 10107.

29. Bursi, L.; Calzolari, A.; Corni, S.; Molinari, E. Quantifying the Plasmonic Character of Optical Excitations in Nanostructures. ACS Photonics 2016, 3, 520-525. 
30. Fitzgerald, J. M.; Azadi, S.; Giannini, V. Quantum Plasmonic Nanoantennas. Phys. Rev. B 2017, 95, 235414.

31. Zhang, R.; Bursi, L.; Cox, J. D.; Cui, Y.; Krauter, C. M.; Alabastri, A.; Manjavacas, A.; Calzolari, A.; Corni, S.; Molinari, E.; Carter, E. A.; de Abajo, F. J. G.; Zhang, H.; Nordlander, P. How to Identify Plasmons from the Optical Response of Nanostructures. ACS Nano 2017, 11, $7321-7335$.

32. Townsend, E.; Bryant, G. W. Plasmonic Properties of Metallic Nanoparticles: The Effects of Size Quantization. Nano Lett. 2011, 12, 429-434.

33. Andersen, K.; Jacobsen, K. W.; Thygesen, K. S. Spatially Resolved Quantum Plasmon Modes in Metallic Nano-Films from First-Principles. Phys. Rev. B 2012, 86, 245129.

34. Townsend, E.; Debrecht, A.; Bryant, G. W. Approaching the Quantum Limit for Nanoplasmonics. J. Mater. Res. 2015, 30, 2389-2399.

35. Casanova, D.; Matxain, J. M.; Ugalde, J. M. Plasmonic Resonances in the Al13-Cluster: Quantification and Origin of Exciton Collectivity. J. Phys. Chem. C 2016, 120, 12742-12750.

36. He, Y.; Zeng, T. First-Principles Study and Model of Dielectric Functions of Silver Nanoparticles. J. Phys. Chem. C 2010, 114, 18023-18030.

37. Malola, S.; Lehtovaara, L.; Enkovaara, J.; Häkkinen, H. Birth of the Localized Surface Plasmon Resonance in Monolayer-Protected Gold Nanoclusters. ACS Nano 2013, 7, $10263-$ 10270.

38. Rossi, T. P.; Kuisma, M.; Puska, M. J.; Nieminen, R. M.; Erhart, P. Kohn-Sham Decomposition in Real-Time Time-Dependent Density-Functional Theory: An Efficient Tool for Analyzing Plasmonic Excitations. J. Chem. Theory Comput. 2017, 13, 4779-4790.

39. Yabana, K.; Bertsch, G. F. Time-dependent Local-Density Approximation in Real Time. Phys. Rev. B 1996, 54, 4484-4487. 
40. Thongrattanasiri, S.; Manjavacas, A.; Garcia de Abajo, F. J. Quantum Finite-Size Effects in Graphene Plasmons. ACS Nano 2012, 6, 1766-1775.

41. Manjavacas, A.; Marchesin, F.; Thongrattanasiri, S.; Koval, P.; Nordlander, P.; SanchezPortal, D.; Garcia de Abajo, F. J. Tunable Molecular Plasmons in Polycyclic Aromatic Hydrocarbons. ACS Nano 2013, 7, 3635-3643.

42. Guidez, E. B.; Aikens, C. M. Origin and TDDFT Benchmarking of the Plasmon Resonance in Acenes. J. Phys. Chem. C 2013, 117, 21466-21475.

43. Kuisma, M.; Ojanen, J.; Enkovaara, J.; Rantala, T. T. Kohn-Sham Potential with Discontinuity for Band Gap Materials. Phys. Rev. B 2010, 82, 115106.

44. Kuisma, M.; Sakko, A.; Rossi, T. P.; Larsen, A. H.; Enkovaara, J.; Lehtovaara, L.; Rantala, T. T. Localized Surface Plasmon Resonance in Silver Nanoparticles: Atomistic FirstPrinciples Time-Dependent Density-Functional Theory Calculations. Phys. Rev. B 2015, 91, 115431.

45. Kramida, A.; Yu. Ralchenko,; Reader, J.; and NIST ASD Team, NIST Atomic Spectra Database (ver. 5.6.1), [Online]. Available: https://physics.nist.gov/asd [2019, February 10]. National Institute of Standards and Technology, Gaithersburg, MD., 2018.

46. Clementi, E.; Raimondi, D. L.; Reinhardt, W. P. Atomic Screening Constants from SCF functions. II. Atoms with 37 to 86 Electrons. J. Chem. Phys. 1967, 47, 1300-1307.

47. Yannouleas, C.; Broglia, R. A.; Brack, M.; Bortignon, P. F. Fragmentation of the Photoabsorption Strength in Neutral and Charged Metal Microclusters. Phys. Rev. Lett. 1989, 63, 255-258.

48. Nayyar, N. Optical and Magnetic Properties of Nanostructures. Ph.D. thesis, University of Central Florida, 2014.

49. Hohenberg, P.; Kohn, W. Inhomogeneous Electron Gas. Phys. Rev. 1964, 136, B864-B871. 
50. Kohn, W.; Sham, L. J. Self-Consistent Equations Including Exchange and Correlation Effects. Phys. Rev. 1965, 140, A1133-A1138.

51. Yan, J.; Jacobsen, K. W.; Thygesen, K. S. First-Principles Study of Surface Plasmons on $\operatorname{Ag}(111)$ and H/Ag(111). Phys. Rev. B 2011, 84, 235430.

52. Yan, J.; Jacobsen, K. W.; Thygesen, K. S. Conventional and Acoustic Surface Plasmons on Noble Metal Surfaces: A Time-Dependent Density Functional Theory Study. Phys. Rev. B 2012, 86, 241404.

53. Mortensen, J. J.; Hansen, L. B.; Jacobsen, K. W. Real-Space Grid Implementation of the Projector Augmented Wave Method. Phys. Rev. B 2005, 71, 035109.

54. Enkovaara, J.; Rostgaard, C.; Mortensen, J. J.; Chen, J.; Dułak, M.; Ferrighi, L.; Gavnholt, J.; Glinsvad, C.; Haikola, V.; Hansen, H. A.; Kristoffersen, H. H.; Kuisma, M.; Larsen, A. H.; Lehtovaara, L.; Ljungberg, M.; Lopez-Acevedo, O.; Moses, P. G.; Ojanen, J.; Olsen, T.; Petzold, V. et al. Electronic Structure Calculations with GPAW: A Real-Space Implementation of the Projector Augmented-Wave Method. J. Phys.: Condens. Matter 2010, 22, 253202.

55. Walter, M.; Häkkinen, H.; Lehtovaara, L.; Puska, M.; Enkovaara, J.; Rostgaard, C.; Mortensen, J. J. Time-Dependent Density-Functional Theory in the Projector AugmentedWave Method. J. Chem. Phys. 2008, 128, 244101.

56. Blöchl, P. E. Projector Augmented-Wave Method. Phys. Rev. B 1994, 50, 17953-17979.

57. Larsen, A. H.; Mortensen, J. J.; Blomqvist, J.; Castelli, I. E.; Christensen, R.; Dułak, M.; Friis, J.; Groves, M. N.; Hammer, B.; Hargus, C.; Hermes, E. D.; Jennings, P. C.; Jensen, P. B.; Kermode, J.; Kitchin, J. R.; Kolsbjerg, E. L.; Kubal, J.; Kaasbjerg, K.; Lysgaard, S.; Maronsson, J. B. et al. The Atomic Simulation Environment-A Python Library for Working with Atoms. J. Phys.: Condens. Matter 2017, 29, 273002. 
58. Larsen, A. H.; Vanin, M.; Mortensen, J. J.; Thygesen, K. S.; Jacobsen, K. W. Localized Atomic Basis Set in the Projector Augmented Wave Method. Phys. Rev. B 2009, 80, 195112.

59. Frisch, M. J.; Trucks, G. W.; Schlegel, H. B.; Scuseria, G. E.; Robb, M. A.; Cheeseman, J. R.; Montgomery, J. A., Jr.; Vreven, T.; Kudin, K. N.; Burant, J. C.; Millam, J. M.; Iyengar, S. S.; Tomasi, J.; Barone, V.; Mennucci, B.; Cossi, M.; Scalmani, G.; Rega, N.; Petersson, G. A.; Nakatsuji, H. et al. Gaussian 03, Revision D.01. Gaussian, Inc., Wallingford, CT, 2004.

60. Becke, A. D. Density-Functional Thermochemistry. III. The Role of Exact Exchange. J. Chem. Phys. 1993, 98, 5648-5652.

61. Perdew, J. P.; Yue, W. Accurate and Simple Density Functional for the Electronic Exchange Energy: Generalized Gradient Approximation. Phys. Rev. B 1986, 33, 8800.

62. Perdew, J. P. Electronic Structure of Solids, Eds. P. Ziesche and H. Eschrig. Berlin: Academic, 1991.

63. Hay, P. J.; Wadt, W. R. Ab initio Effective Core Potentials for Molecular Calculations. Potentials for K to Au Including the Outermost Core Orbitals. J. Chem. Phys. 1985, 82, 299-310. 